\title{
A SIMPLE DERIVATION OF THE LONG WAVELENGTH EDGE RADIATION FROM A BENDING MAGNET
}

\author{
M. Castellano \# \\ INFN - Laboratori Nazionali di Frascati - CP 13 - 00044 Frascati - ITALY
}

\begin{abstract}
A simple derivation of the "edge radiation" emitted by an electron beam going through a bending magnet in the directions of its entrance and exit from the magnet itself is presented. This radiation is characterized by wavelengths much longer than the synchrotron radiation critical wavelength in the same magnet.

The far field radiation emitted by an electron following a curved trajectory inside a finite bending magnet can be approximated with any required accuracy by that produced by an electron following a segmented trajectory made of straight lines with sudden change of the velocity direction. The radiation field amplitude produced by a velocity direction change is that of a "prompt" bremsstrahlung in which the velocity module remains constant. The total amplitude is the sum of all the bremsstrahlung amplitudes with their relative phase difference.

For long wavelengths and around the direction of entrance or exit from the magnet, only few terms are required to give a good description of the radiation as compared with exact calculations
\end{abstract}

\section{INTRODUCTION}

The so-called "edge radiation" is emitted by an electron beam going through a bending magnet in the directions of its entrance and exit from the magnet itself. It is characterized by wavelengths much longer than the synchrotron radiation critical wavelength in the same magnet. It is considered for possible scientific applications because may be brighter than the analogous radiation emitted along the central trajectory inside the bending magnets.

It has been often assumed that this radiation could depend from the behavior of the magnet fringing field. I will demonstrate that it is only the standard synchrotron radiation from a finite length magnet.

R.A. Bosch has given a simple interpretation [1] that has some validity in the very long wavelength limit and can be considered a "zero order" approximation.

I will give a more general derivation, valid for all wavelengths and emission angles, which coincides with standard synchrotron radiation at short wavelengths.

The starting point is the observation that the far field radiation emitted by an electron following a curved trajectory inside a finite bending magnet can be approximated with any required accuracy by that produced by an electron following a segmented trajectory made of straight lines with sudden change of the velocity direction.

\#michele.castellano@lnf.infn.it
The radiation field amplitude produced by a velocity direction change is that of a "prompt" bremsstrahlung in which the velocity module remains constant. The total amplitude is the sum of all the bremsstrahlung amplitudes with their relative phase difference.

For long wavelengths and around the direction of entrance or exit from the magnet, only few terms are required to give a good description of the radiation as compared with exact calculations [2]. The Bosch approximation consists in considering only the first term.

\section{THEORETICAL CONSIDERATIONS}

The starting point is the expression of the radiation intensity emitted by a charged particle in an arbitrary accelerated motion [3]

$$
\frac{\mathrm{dI}^{2}}{\mathrm{~d} \omega \mathrm{d} \Omega}=\frac{\mathrm{e}^{2}}{4 \pi^{2} \mathrm{c}}\left|\int_{-\infty}^{\infty} \frac{\underline{\mathrm{n}} \times[(\underline{\mathrm{n}}-\underline{\beta}) \times \underline{\beta}]}{(1-\underline{\beta} \cdot \underline{\mathrm{n}})^{2}} \mathrm{e} \mathrm{i \omega (1- \underline { \textrm {n } } \cdot \frac { \mathrm { r } ( \mathrm { t } ) } { \mathrm { c } } )} \mathrm{dt}\right|^{2}
$$

in which $\underline{n}$ is the observation direction and $\underline{\beta}$ the particle velocity.

For a particle travelling only once trough a finite length bending magnet, the integral will be performed between the time of its entrance $t_{o}$ and the time of its exit $t_{f}$.

We can always subdivide the integral as a sum of integrals on smaller time intervals

$$
\frac{\mathrm{dI}^{2}}{\mathrm{~d} \omega \mathrm{d} \Omega}=\frac{\mathrm{e}^{2}}{4 \pi^{2} \mathrm{c}}\left|\sum_{1}^{\mathrm{f}} \int_{\mathrm{t}_{\mathrm{i}-1}}^{\mathrm{t}_{\mathrm{i}}} \frac{\underline{\mathrm{n}} \times[(\underline{\mathrm{n}}-\underline{\beta}) \times \underline{\beta}]}{(1-\underline{\beta} \cdot \underline{\mathrm{n}})^{2}} \mathrm{e}^{\mathrm{i} \omega\left(1-\underline{\mathrm{n}} \cdot \underline{\mathrm{r} \cdot(\mathrm{t})} \frac{\mathrm{c}}{\mathrm{c}}\right)} \mathrm{dt}\right|^{2}
$$

For sufficient short time intervals and long wavelength, we can assume that the phase factor is constant for each integral, so that we can perform an analytical evaluation

$$
\frac{\mathrm{dI}^{2}}{\mathrm{~d} \omega \mathrm{d} \Omega}=\frac{\mathrm{e}^{2}}{4 \pi^{2} \mathrm{c}}\left|\sum_{1}^{\mathrm{f}} \mathrm{e}^{\mathrm{i} \phi_{\mathrm{k}}}\left[\frac{\underline{\mathrm{n}} \times \underline{\beta}}{(1-\underline{\mathrm{n}} \cdot \underline{\beta})^{2}}\right]_{\mathrm{k}-1}^{\mathrm{k}}\right|^{2}
$$

This corresponds to approximate the curved path of the particle with a segmented trajectory, in which the particle velocity, constant in module, changes suddenly its direction. 
The radiation amplitude for each change of direction can be written as that of a particle in uniform motion suddenly stopped, plus that of a particle starting with constant velocity in the new direction. The two amplitudes have opposite sign due to the different accelerations.

This approach of "prompt bremsstrahlung" has proven its efficacy in many radiation processes, from standard transition radiation to undulator transition radiation [4].

For our purpose, we can rearrange the terms of the sum in (1)

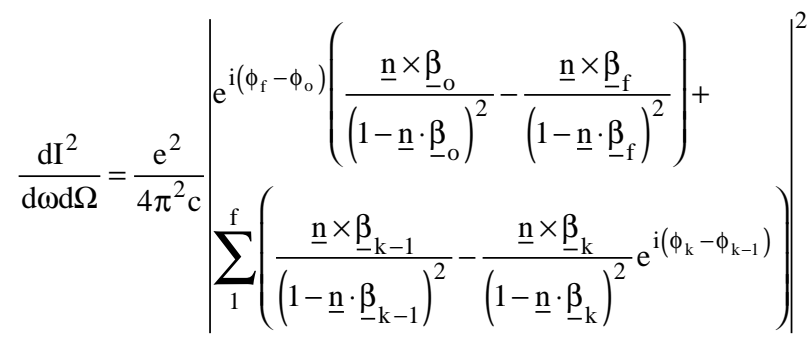

The first two terms in (2) represent, respectively, the radiation field amplitude produced by a particle that, moving at constant velocity, stops suddenly at the entrance of the bending magnet, and that of a particle at rest that starts moving in uniform motion at the exit of the magnet. Each of these amplitudes, that are often found in radiation phenomena, as Optical Transition Radiation, produce a radiation angular distribution with a maximum intensity at an angle equal to $1 / \gamma$ with respect to the particle velocity, $\gamma$ being the particle relativistic factor.

If the bending angle caused by the magnet is much larger than $1 / \gamma$, the two amplitudes do not interfere, independently of the wavelength, and we have two separate sources each with the properties of a prompt bremsstrahlung. This is the approximation introduced in [1]. In this case the radiation is distributed symmetrically around the direction of entrance and exit of the beam from the magnet, and has a flat spectrum. Exact numerical calculations [2] show that this approximation has some validity only for very long wavelengths, and is not able to give the radiation intensity at larger angles, where the other terms in (2) are much more important.

These terms also are not new in the radiation panorama. Each element of the sum represents the field amplitude produced by a particle starting, from rest, at constant velocity in a given direction and then stopping after a finite path length. In the segmented trajectory model, this represent the radiation amplitude produced by the particle in a single rectilinear segment. This amplitude has been studied in transition radiation emission as the interference between two radiating foils, and, equivalently, but less known, as an under threshold Cherenkov radiation.

To better understand the effects of these terms on the total amplitude, we will analyze the behavior of one of them in the horizontal plane, where its contribution is more sensible.

We have two equal amplitude of opposite sign separated by a phase difference that can be written as

$$
\phi=\frac{2 \pi \mathrm{d}}{\lambda}(1-\beta \cos \theta)
$$

in which $d$ is the distance traveled by the particle and $\theta$ is the observation angle with respect to the particle velocity.

The intensity distribution produced by a single segment of the trajectory is given by

$$
\frac{\mathrm{dI}^{2}}{\mathrm{~d} \omega \mathrm{d} \Omega}=\frac{\mathrm{e}^{2}}{\mathrm{c}}\left(\frac{\mathrm{d}}{\lambda}\right)^{2} \sin ^{2} \theta\left(\frac{\sin \frac{\phi}{2}}{\frac{\phi}{2}}\right)^{2}
$$

It is clear that the smaller is the ratio $d / \lambda$, the lesser is the intensity, but spanning a larger angle range. This must be kept in mind when deciding how segmenting the trajectory and how many terms of the sum in (2) should be considered.

\section{NUMERICAL SIMULATIONS}

To demonstrate the effectiveness of this approach, I have calculated the radiation angular distribution in the horizontal plane for a real case, i.e. the Super-ACO storage ring, for which exact calculations and measurements exist [2].

The machine parameters relevant for this calculation are summarized in Table 1.

Table 1 - Relevant parameters of Super-ACO

\begin{tabular}{|c|c|c|}
\hline Energy & 800 & $\mathrm{MeV}$ \\
\hline Bending angle & $\pi / 4$ & $\mathrm{rad}$ \\
\hline Curvature radius & 1.7 & $\mathrm{~m}$ \\
\hline
\end{tabular}

The large bending angle prevents any interference between amplitudes at the entrance and exit of the magnet, for all reasonable wavelength value, so that the first two amplitudes in (2) can be considered separately.

In the following I will show some example of the radiation angular distribution along the exit direction from the magnet. In this case the first term in (2) can be neglected. In Fig. 1 the effect of a single bremsstrahlung amplitude (second term in (2)) is shown. This corresponds to the "zero length" model presented in [1]. Intensity and angular distribution of the radiation do not depend from wavelength. It must be noted that for practical reasons the intensity is given in arbitrary units, but the scale is the same for all the pictures, which can be directly compared.

Positive angles are towards the inner part of the curved trajectory. 


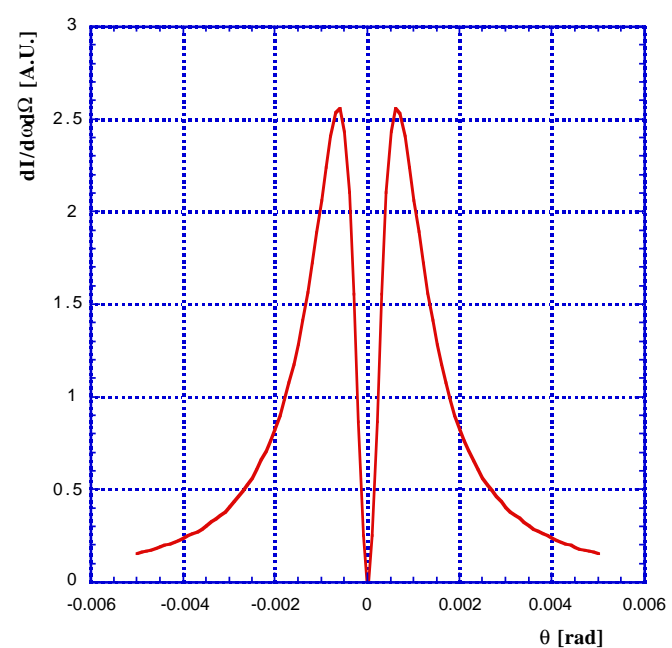

Fig 1 - Edge radiation angular distribution produced by a single bremsstrahlung amplitude

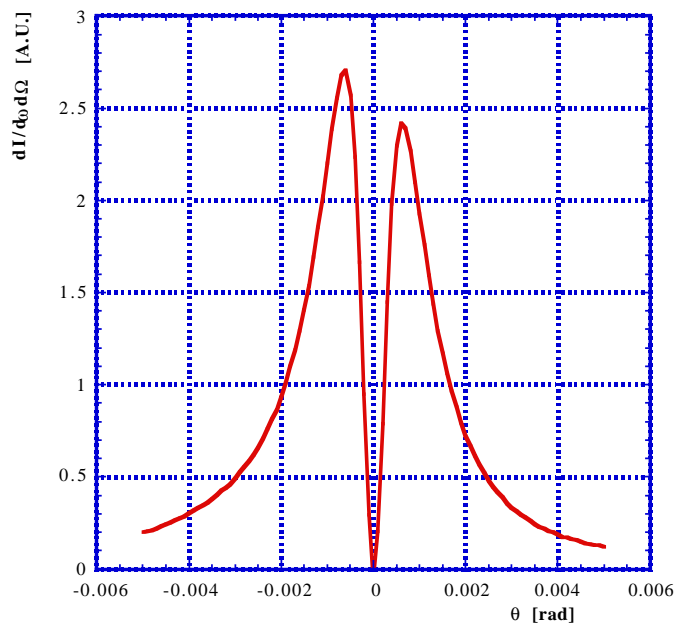

Fig 2 - Angular distribution of edge radiation at $500 \mu \mathrm{m}$

In Fig. 2 it is represented the same distribution for a radiation wavelength of $500 \mu \mathrm{m}$ obtained from (2) approximating the curved trajectory as 15 linear segments, but considering only the contribution of the last three.

The asymmetry between the two lobes is now evident, and can be compared with that calculated in [2].

Even if based on a small number of terms, this result is accurate, compared to exact evaluations, to better than $1 \%$ on the peaks and to better than $5 \%$ on the tails.

Increasing the number of segments and the number of terms considered in (2), the intensity distribution at shorter wavelength can be computed with the same accuracy. As it is shown if Fig. 3, the asymmetry of the lobes increases and the distribution approaches the flat behavior of the "standard" synchrotron radiation.

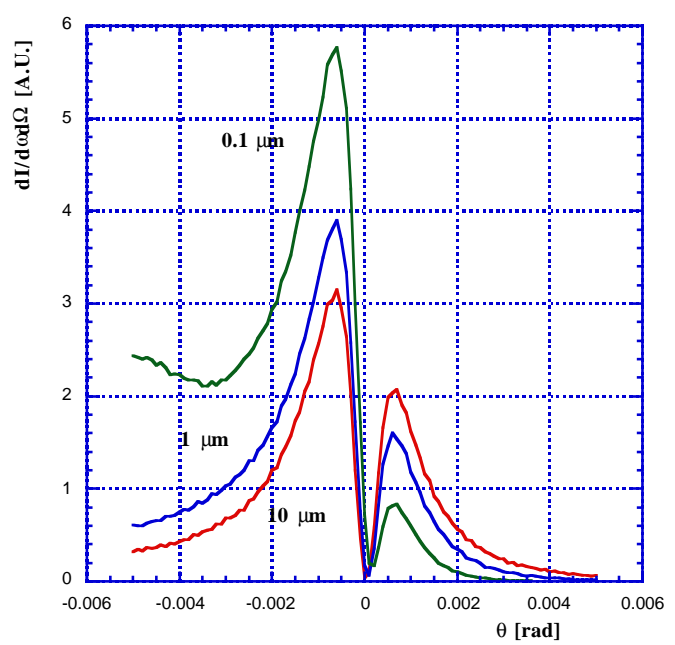

Fig 3 - Edge radiation angular distribution at shorter wavelength

\section{CONCLUSIONS}

The approximation of the trajectory inside a bending magnet as a sum of straight segments allows an intuitive and simple evaluation of the radiation emitted along the directions of entrance and exit from the magnet. In the case of long wavelengths an accurate result can be obtained by a simple expression. The use of prompt bremsstrahlung amplitudes allows also to consider the interference with other possible source of radiation along the straight section, due to insertion devices or even the mirror used to extract the edge radiation, in a simple way.

\section{REFERENCES}

[1] R.A. Bosch, Il Nuovo Cimento 20D, 483, (1998)

[2] P. Roy et al., Il Nuovo Cimento 20D, 415, (1998)

[3] J.D. Jackson, Classical Electrodynamics, J. Wiley \& sons

[4] M. Castellano, Nucl. Instr. and Meth. in Phys. Res. A391, 375 (1997) 